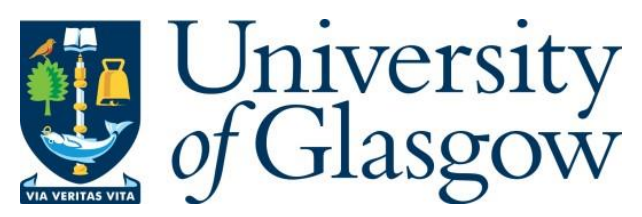

Krämer, S. (2014) Implicit commitment in theory choice. Synthese, 191(10), pp. 21472165.

There may be differences between this version and the published version. You are advised to consult the publisher's version if you wish to cite from it.

http://eprints.gla.ac.uk/140556/

Deposited on: 4 May 2017

Enlighten - Research publications by members of the University of Glasgow http://eprints.gla.ac.uk 


\title{
Implicit Commitment in Theory Choice
}

\author{
STEPHAN KRÄMER
}

[This is a penultimate version. Please quote only from the published version.

The final publication is available at http://link.springer.com.]

\begin{abstract}
The proper evaluation of a theory's virtues seems to require taking into account what the theory is indirectly or implicitly committed to, in addition to what it explicitly says. Most extant proposals for criteria of theory choice in the literature spell out the relevant notion of implicit commitment via some notion of entailment. I show that such criteria behave implausibly in application to theories that differ over matters of entailment. A recent defence by Howard Peacock of such a criterion against this objection is examined and rejected. I go on to a develop a better proposal on which, roughly speaking, a theory is counted committed to a claim if and only if its best fully explicit extension is explicitly committed to the claim. Such extensions in turn are evaluated by ordinary standards of theory choice adapted to the case of theories assumed to articulate their intended content in a fully explicit fashion.
\end{abstract}

Keywords: Commitment; Theory Choice; Entailment; Incomparability; Logical Disagreement

\section{Introduction}

According to a widely held view, rational choice between competing theories is informed at least in part by an assessment of the extent to which the theories in question exemplify certain (putative) theoretical virtues. Examples of these are strength, explanatory power, compatibility with what we know, and certain forms of simplicity such as ontological parsimony. ${ }^{1}$ For some of these virtues, it appears that to evaluate to what extent a theory exhibits them, we must look beyond the explicit contents of the sentences making up the theory. For instance, if I know it to be false that there are no tables, a theory can be

\footnotetext{
${ }^{1}$ The view is widely but of course not universally shared; alternative approaches to theory choice are possible, such as Popperian corroborationism, probabilism, or decision theory, on which putative theoretical virtues may have no obvious role to play. Thanks here to an anonymous referee.
} 
incompatible with that piece of knowledge without containing a sentence that means there are no tables. The obvious-seeming conclusion is that we must also consider what a given theory entails, in some suitable sense of 'entails'. I will argue that on any ordinary notion of entailment, this conclusion is false: general criteria for theory choice invoking what the theories entail do not conform to how we actually, and reasonably, choose between competing theories. I go on to present an alternative criterion that fares better.

For simplicity, and because it is a (putative) theoretical virtue that has been getting a lot of attention in recent debates, in what follows I shall focus on ontological parsimony. The important points in the discussion generalize to the assessment of theories with respect to any other theoretical virtue that similarly seems to depend on what a theory entails. By 'theory' I shall understand any set of sentences (of some interpreted language). I will not require that a set of sentences be closed under logical consequence in order to count as a theory. ${ }^{2}$ There are two reasons for this. In practice, what we want to compare for ontological parsimony are the kinds of things we find written down in a book or an article, which seem more naturally modelled by the set of sentences that are thus written down than by the closure of such a set under logical consequence. Moreover, if we were to require closure under logical consequence, we could not adequately represent as a (formal) theory any view that endorses a mistaken logic.

The view that ontological parsimony is a theoretical virtue is captured in the principle of Occam's Razor, which says that given two competing theories, the one (if either) which carries more substantial ontological commitments is in this respect at a comparative disadvantage; other things being equal, we should prefer its rival. If other things are not equal, we have to decide whether the cost of the additional ontology is outweighed by benefits the theory offers in other respects. In the general case, application of Occam’s Razor to a given pair of theories requires that we determine what ontological commitments each of them carries, and whether those carried by one are more substantial than those carried by the other. ${ }^{3}$ I have nothing to say about how questions of the second sort are to be answered; for our purposes we can make do with examples where the answer to that question is uncontentious.

\footnotetext{
${ }^{2}$ A set of sentences is closed under logical consequence iff it includes every sentence logically entailed by it.

${ }^{3}$ Appeals to Occam's Razor are generally relative to some phenomena to be explained, or to some specific subject matter that the theories are supposed to be theories of. We shall usually leave the specification of subject matter or phenomena implicit. (Thanks here to an anonymous referee.)
} 
My focus will be entirely on the conditions under which a theory carries a given ontological commitment. $^{4}$

In order for a theory which carries a commitment to Fs to be true, that commitment must be satisfied by the world. Everyone agrees that for some sense of 'there is/are', frequent and familiar at least in philosophical discussion, a commitment to Fs is satisfied just in case, in that sense of 'there is/are', there are Fs. I shall frequently use 'there is/are' on the understanding that it expresses that sense. Everyone can then agree that a theory's including among its members a sentence which means there are Fs is a sufficient condition for the theory's carrying ontological commitment to Fs. If a theory is committed to Fs for just this reason, I shall say that it is explicitly ontologically committed to Fs:

(Df. EOC) A theory T is explicitly ontologically committed to Fs $\leftrightarrow d f$

$\mathrm{T}$ includes some sentence that means there are Fs.

Commitments which are not explicit we will accordingly call 'implicit'.

(Df. IOC) A theory T is implicitly ontologically committed to Fs $\leftrightarrow_{d f}$

T is ontologically committed to Fs \&

$\mathrm{T}$ is not explicitly ontologically committed to Fs.

By our assumptions, explicit commitment entails commitment: if a theory is explicitly ontologically committed to Fs, then it is ontologically committed to $\mathrm{Fs}^{5}$

To obtain a criterion of commitment, we now need to answer the question under what conditions a theory is implicitly committed to Fs. It seems extremely plausible that the answer to this question is not never. Some toy examples may suffice to make the point. ${ }^{6}$ For instance, the theory $\{$ ' $\exists x x$ is a number) \& $(\exists x x$ is a number $)$ ' $\}$ is not explicitly committed to numbers - or indeed to anything at all -, for its only member does not mean there are numbers. It seems obvious, however, that the theory is committed to numbers. Otherwise, it would turn

\footnotetext{
${ }^{4}$ It is worth emphasizing that the interest of the main points of my paper therefore does not depend on Occam's Razor but only on the weaker claim that what a theory is committed to is relevant to theory choice.

${ }^{5}$ In order to decide what a given theory is explicitly committed to, we need to know which of its expressions mean there is (in the ontologically important sense). I assume throughout the orthodox view that the ordinary first-order existential quantifier ' $\exists x$ ' does; nothing of substance hinges on that assumption. (For recent criticisms of the assumption, see e.g. Azzouni 2007 and Fine 2009.)

${ }^{6}$ For related arguments for the same point, cp. e.g. Peacock 2011, pp. $81 \mathrm{ff}$.
} 
out as more (ontologically ${ }^{7}$ ) parsimonious than the theory resulting from adding to it the sentence ' $\exists x x$ is a number' which seems bizarre. Similarly, it seems that $\{$ ' $\exists x x$ is a vixen' $\}$ is committed to foxes, and that $\{$ ' $\exists x x$ is a composite object' $\}$ is committed to proper parts, but neither theory is explicitly so committed. The obvious idea is to adopt, on some suitable notion of entailment, a criterion of Ontological Commitment by Entailment:

(OCE) A theory $\mathrm{T}$ is committed to Fs iff $\mathrm{T}$ entails that there are Fs.

For want of a better term, I shall call criteria of this shape entailment-based. It must be emphasized that, in spite of the connotations to the contrary of the word 'entailment-based', in order for a criterion to count as entailment-based, it is not sufficient that the criterion invoke, and give some important role to, a notion of entailment. In my terminology, in order to count as entailment-based, a criterion must give a very specific role to a notion of entailment, namely the role identified by (OCE). We may accordingly distinguish between two kinds of possible non-entailment-based criteria: ones that do not make use of a notion of entailment at all, and ones that do make use of such a notion, but employ it in a subtly different role. The next two sections argue that no entailment-based criterion is acceptable. The final section develops and defends an alternative criterion, belonging to the second kind of non-entailmentbased criteria.

\section{Entailment-based Criteria and the Problem of Incomparability}

In a rough sketch, the basic problem for entailment-based criteria is that they cannot be nonquestion-beggingly applied to pairs of theories that (explicitly or implicitly) disagree on matters of entailment. As a result, they predict that rational, non-question-begging choice between such pairs is impossible. In many relevant cases however, this seems clearly wrong.

First, consider the criterion obtained through a simple modal explication of entailment (OCM) A theory $\mathrm{T}$ is ontologically committed to Fs iff necessarily, if $\mathrm{T}$ is true (on its actual interpretation), then there are Fs.

This criterion, or some minor variation of it, has been accepted by various authors including e.g. Frank Jackson (1989: 192), Alex Oliver (1996, p. 60), Michael Devitt (1980, p. 435), and

\footnotetext{
${ }^{7}$ When in the sequel I speak of parsimony, unless otherwise noted, I mean ontological parsimony.
} 
Peter Simons (1997, p. 262). More recently, it has been defended in some detail by Howard Peacock (2011, esp. pp. 84, 90, 103f).

As has been noted by at least some of its proponents, (OCM) yields some results that at first glance seem extremely implausible. First, if, for instance, sets exist necessarily, then on (OCM), every theory is ontologically committed to sets, even if it explicitly denies that there are sets. Second, on (OCM), every theory which is necessarily false is maximally committed, i.e. committed to $F$ s, for arbitrary ' $F$ ' ${ }^{8}$ Although I consider this a very significant problem for (OCM), it is not the problem I want to focus on. For my purposes, therefore, we need not decide whether it has a convincing solution. ${ }^{9}$

The problem I am interested in can be illustrated by the following example. Let $\mathrm{T}_{\mathrm{UM}}$ (Universalism, Modal) be the theory including 'there are at least two objects' and 'necessarily, any two objects compose a further object', and $\mathrm{T}_{\mathrm{NM}}$ (Nihilism, Modal) be the theory including 'there are at least two objects', and 'necessarily, no two objects compose a further object'. Suppose Joe wants to decide which of these theories he should accept. Assume further that as part of this inquiry, Joe tries to find out which of the theories is more parsimonious. Then we find that unfortunately, (OCM) renders it impossible for Joe to do this in a non-questionbegging way. The crucial points are as follows.

Firstly, in the context of this inquiry, certain claims are question-begging in the sense that it would be question-begging for Joe to employ them as premises in his reasoning about the respective merits of $\mathrm{T}_{\mathrm{UM}}$ and $\mathrm{T}_{\mathrm{NM}}$. To a rough approximation, these are all and only those claims which straightforwardly entail the truth or falsity of either of the theories in question.

Secondly, in order to determine, on the basis of (OCM), which of $\mathrm{T}_{\mathrm{UM}}$ and $\mathrm{T}_{\mathrm{NM}}$ is more parsimonious, Joe needs to employ premises concerning their modal status. In particular, he needs to employ either the premise that $\mathrm{T}_{\mathrm{UM}}$ is possibly true, or the premise that it is not possibly true, and he needs to employ either the premise that $\mathrm{T}_{\mathrm{NM}}$ is possibly true, or the premise that it is not possibly true. For recall that on (OCM), any theory which is not

\footnotetext{
${ }^{8}$ If $\mathrm{T}$ is necessarily false, whatever one puts for ' $F$ ', the right-hand side of (OCM) is vacuously satisfied since the antecedent ' $\mathrm{T}$ is true (on its actual interpretation)' of the embedded conditional is necessarily false. So if $\mathrm{T}$ is necessarily false, whatever one puts for ' $F$ ', (OCM) implies that $\mathrm{T}$ is committed to $F$ s.

${ }^{9}$ Peacock directly discusses the first problem (cf. Peacock 2011, 90ff), and his response to the problem I am about to discuss is at least suggestive of a way to deal with the second. I discuss this response in section 3 below.
} 
possibly true is maximally committed and therefore not more parsimonious than any theory whatsoever. So Joe can count neither theory more parsimonious than the other as long as he has not ruled out that the theory is necessarily false. ${ }^{10}$

Thirdly, because of what $\mathrm{T}_{\mathrm{UM}}$ and $\mathrm{T}_{\mathrm{NM}}$ say, these premises are question-begging. For if $\mathrm{T}_{\mathrm{NM}}$ is possibly true, it is possible that there are two objects that do not compose, so it is not necessary that any two objects compose, so $\mathrm{T}_{\mathrm{UM}}$ is false. Similarly, if $\mathrm{T}_{\mathrm{UM}}$ is possibly true, then it is possible that there are two objects that compose, so it is not necessary that no two objects compose, so $\mathrm{T}_{\mathrm{NM}}$ is false. Moreover, these bits of modal reasoning are as straightforward and uncontentious as one could wish them to be. So, given (OCM), Joe cannot determine which of the two theories is more parsimonious without relying on premises that are question-begging in the given context of theory choice. Note that the point is not that, contra (OCM), necessarily false theories are not maximally committed. Nothing I say assumes this. The point is that to ascertain which of two theories is more parsimonious, we need to know whether one of them is maximally committed, and in the case discussed, using (OCM), we cannot figure that out without first deciding which theory is true.

The source of the problem is that application of (OCM) to $\mathrm{T}_{\mathrm{UM}}$ and $\mathrm{T}_{\mathrm{NM}}$ requires taking a stand on certain questions of modal entailment, in particular on what $\mathrm{T}_{\mathrm{UM}}$ and $\mathrm{T}_{\mathrm{NM}}$ modally entail, while at the same time these theories themselves implicitly take opposite stands on exactly those questions of modal entailment. A version of the problem arises even for the non-modal counterparts $\mathrm{T}_{\mathrm{U}}$ and $\mathrm{T}_{\mathrm{M}}$ of $\mathrm{T}_{\mathrm{UM}}$ and $\mathrm{T}_{\mathrm{NM}}$ when we assume that these are necessarily true if true, and necessarily false if false, and that one of them is true. In comparing the ontological costs of $T_{U}$ and $T_{M}$, Joe needs to employ premises saying whether the theories are possibly true. Moreover, if he is to arrive at a correct assessment of the theories' ontological costs, the premises he employs had better be true. Since both theories are necessary if true, and since each is necessarily false if the other is necessarily true, exactly one of them is necessarily false and thus maximally committed. So in order to correctly determine,

\footnotetext{
${ }^{10}$ It is worth stressing that the point does not assume that the result that necessarily false theories are maximally committed is implausible; indeed, we could make essentially the same point without appeal to that result. For instance, we might add to $\mathrm{T}_{\mathrm{UM}}$ its standard modal logical consequence 'necessarily, if no two objects compose, then any two objects compose', which has to be assumed false if we are to maintain that $\mathrm{T}_{\mathrm{NM}}$ does not entail that any two objects compose and accordingly is not committed to composite objects. Then to decide whether $\mathrm{T}_{\mathrm{NM}}$ is committed to composite objects, we need to assume either the falsity of it, or the falsity of $\mathrm{T}_{\mathrm{UM}}$.
} 
in accordance with (OCM), the commitments of $T_{U}$ and $T_{M}$, Joe needs to employ a premise which ascribes necessary falsity to one of the theories, making it question-begging.

These results speak strongly against (OCM). It is important to be precise about exactly why they do. The crucial point is not that (OCM) yields false ascriptions of commitment, or that it may be contentious what ascriptions of commitment it implies. Nor is the point that there is necessarily something wrong with evaluating a theory in a question-begging way, rejecting it on the grounds that it is ruled out by a claim one independently considers established. ${ }^{11}$ The point is, firstly, that sometimes when we wish to choose between two theories, we do in fact start from a neutral standpoint. In such situations, we do not have any independent beliefs that would rule out one of the theories. The result just presented shows that in that situation, for pairs of theories like $\mathrm{T}_{\mathrm{UM}}$ and $\mathrm{T}_{\mathrm{NM}}$, we also do not have the sorts of beliefs required to even work out which is more parsimonious according to (OCM). Secondly, even if we do happen to have independent beliefs that decide against one of the theories considered, it is still an interesting question worthwhile to investigate how the theories compare from a neutral standpoint. So one may reasonably decide to temporarily suspend one's beliefs that decide the case, to then work out which theory seems preferable from the resulting, neutral point of view. Again, the result I presented shows that on (OCM), once one has suspended all question-begging beliefs regarding $\mathrm{T}_{\mathrm{UM}}$ and $\mathrm{T}_{\mathrm{NM}}$, one does not have enough left to work out which is more parsimonious. Moreover the problem cases are not in relevant respects atypical; cases in which each of two rival theories is necessary if true, and thus in which the possible truth of each implies the necessary falsity of the other, are not at all unusual in philosophy. Thus, there are very common and sensible theoretical purposes for which we require a criterion of commitment and for which (OCM) is plainly inadequate. ${ }^{12}$

\footnotetext{
${ }^{11}$ Thanks to an anonymous referee for pressing me on this.

${ }^{12}$ One might wonder if the problem can be circumvented if one assumes that in order not to beg the question against a theory, it is sufficient to consider it an epistemic rather than a metaphysical possibility. (This idea was suggested to me by an anonymous referee.) Unfortunately, I do not think that this will ultimately help. In the case at hand, a full answer to what the theories are committed to must be based, among other things, on a premise that ascribes metaphysically necessary falsity to one of the theories - call that theory T. Now suppose T is an epistemic possibility for me, so that for all I know, T may be true. Then presumably for all I know, T may not be metaphysically impossible. But if nothing I know rules out the metaphysical possibility of T then I cannot appropriately determine T's commitments by making use of the premise that $\mathrm{T}$ is metaphysically impossible.
} 
Of course, in disputes over very basic logical or metaphysical issues, it sometimes really is very difficult, and perhaps sometimes impossible, to evaluate the competing views without begging questions. So does the impossibility of applying (OCM) in a non-questionbegging way to $\mathrm{T}_{\mathrm{UM}}$ and $\mathrm{T}_{\mathrm{NM}}$ simply track a real, genuine impossibility of non-questionbegging comparison? That seems very implausible. Indeed, the incomparability of $\mathrm{T}_{\mathrm{UM}}$ and $\mathrm{T}_{\mathrm{NM}}$ on (OCM) seems entirely artificial in that informally, we do not seem to face any difficulty in determining which of the theories is more parsimonious without first deciding on their truth-values. On the contrary, it seems obvious that $\mathrm{T}_{\mathrm{NM}}$ is more parsimonious, since it does not, whereas $\mathrm{T}_{\mathrm{UM}}$ does, require an ontology of composite objects. ${ }^{13}$ In order to obtain this result, therefore, we do not need to decide what the theories (metaphysically) entail. In particular, in order to obtain the result that $\mathrm{T}_{\mathrm{NM}}$ is not committed to composite objects, we need not assume that it fails to entail the existence of composite objects. Rather, we take $\mathrm{T}_{\mathrm{NM}}$ not to be committed to composite objects exactly because we recognize that any reason for taking $\mathrm{T}_{\mathrm{NM}}$ to entail the existence of composite objects is straightforwardly question-begging.

Numerous other entailment-based criteria of ontological commitment have been presented in the literature that, in effect, replace (OCM)'s appeal to the modal notion of entailment by an appeal to a narrower notion of entailment. ${ }^{14}$ Can we avoid the problem of incomparability by adopting one of these criteria? I think we cannot. The authors mentioned all use notions of entailment wider than logical consequence, and for good reason: otherwise obvious-seeming claims, such as the claim that $\{$ 'there is a vixen' $\}$ is committed to foxes,

\footnotetext{
${ }^{13}$ As the subject matter of the toy theories here compared, we may take the question under what conditions some objects compose a further one; $\mathrm{T}_{\mathrm{NM}}$ offers the more parsimonious answer to that question.

${ }^{14}$ See e.g. the explications of Quinean formulations offered in Cartwright 1954 and Rayo 2007, §2.1, as well as the criterion in terms of a priori entailment in Michael 2008. As argued by e.g. Cartwright 1954 and Parsons 1967, Quine’s own insistence that ontological commitment is an extensional notion, belonging to what he termed the theory of reference (cf. Quine 1961, p. 130f), threatens to leave him with a criterion that fares even worse than (OCM). Here is one way to see the point. If commitment is extensional, then if a theory is committed to Fs, and all and only Fs are Gs, then it is committed to Gs. In particular, if there are no Fs, it is committed to unicorns, sets that are not sets, and more generally self-distinct $G$ s for arbitrary ' $G$ '. So if a theory says there are Fs, then in order to work out what it is committed to, I need to decide whether there are indeed Fs. If I am to choose between the theory in question and a competing one saying that there are no Fs, I cannot do so in a nonquestion-begging way. In contrast to the case of (OCM), here it is not even required that the theories have their status as true or false as a matter of metaphysical necessity, but they may have it contingently.
} 
would have to be rejected. ${ }^{15}$ But even the criterion using logical consequence - call it (OCL) - yields implausible results of incomparability. For the source of incomparability is simply that theories may disagree (explicitly or implicitly) on what entails what, on whatever notion of entailment is employed in the criterion of commitment. Since there is disagreement even over what follows logically from what, there are realistic cases of pairs of theories that are rendered incomparable even by (OCL). ${ }^{16}$ For an example, let $\mathrm{T}_{\text {PARA }}$ be a paraconsistent set theory which includes a classically inconsistent sentence, and which explicitly denies the existence of proper classes. Let $\mathrm{T}_{\text {CLAss }}$ be a classically consistent rival set theory that explicitly affirms the existence of proper classes. Assuming, as I do throughout this paper, that classical logic is correct, on (OCL), $\mathrm{T}_{\text {PARA }}$ is maximally committed. $\mathrm{T}_{\text {CLASS }}$, on the other hand, is not, and so comes out more parsimonious than T $\mathrm{PARA}$. Yet to obtain this result, we need to make use of the premise that $\mathrm{T}_{\text {PARA }}$, due to its containing a logical falsehood, logically entails everything. This premise is question-begging in the context of theory choice between $T_{\text {PARA }}$ and $\mathrm{T}_{\text {CLASS}}$, for it begs the question against $\mathrm{T}_{\text {PARA }}$. As a result, the theories are incomparable on (OCL). This case is not unrealistic: theories relevantly like $T_{\text {PARA }}$ have actual defenders. ${ }^{17}$

As before, the crucial point against (OCL) is not that it implies wrong ascriptions of commitment, but that it renders Occam's Razor useless in application to cases like those mentioned above, when we want to or have to choose non-question-beggingly. And while rarer than those rendered incomparable by (OCM), cases in which competing theories endorse different logics are not so rare that they could be ignored at little or no cost. If a purportedly general principle for theory choice based on ontological parsimony is not applicable to any such cases, then this is a significant drawback of that principle. Finally, the incomparability even of $\mathrm{T}_{\text {CLASS }}$ and $\mathrm{T}_{\mathrm{PARA}}$ seems artificial. Informally, it does not seem as though we have to

\footnotetext{
${ }^{15}$ For arguments that logical entailment is too narrow to serve as the entailment relation in an entailment-based account of commitment, cp. e.g. Searle 1969, pp. 109f, Jackson 1989, p. 193, Michael 2008, p. 50.

${ }^{16}$ Here and in what follows, I assume as correct the view that disagreements over matters of logic are not merely verbal disagreements, and that principled, rational debate about (theories employing) competing logics is possible. Thus, I assume, for instance, that when Graham Priest utters a sentence of the form ' $p$ \& $\neg p$ ' with sincere assertoric intent, he asserts the very claim that is rejected by a classical logician when he utters with sincere assertoric intent the corresponding sentence of the form ' $\neg(p \& \neg p)$ '. So on the view I assume to be correct, the theories $\mathrm{T}_{\text {PARA }}$ and $\mathrm{T}_{\text {CLASS }}$ really do employ different logics that are in competition with each other, and are not just addressing slightly different topics, talking past each other, as it were. For a detailed defence of that view, see e.g. Williamson 2007, ch. 4.

${ }^{17}$ For instance, Priest 2006 advocates a paraconsistent set theory that includes supposedly true contradictions.
} 
first decide what the right logic is before we can assess their comparative ontological parsimony. ${ }^{18}$ It strikes me as less than straightforward to decide which of the theories is more

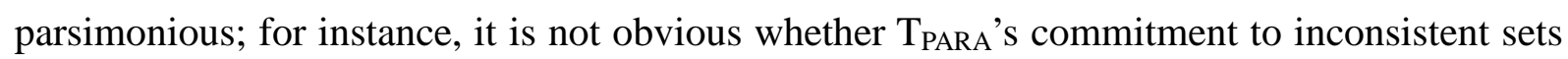
is as substantial as $\mathrm{T}_{\text {CLAss's }}$ commitment to proper classes. But it seems very implausible that $\mathrm{T}_{\text {CLASS }}$ should be more parsimonious for the trivial reason that $\mathrm{T}_{\text {PARA }}$ is maximally unparsimonious. In order to assess their comparative parsimony, therefore, we do not need to decide what the theories logically entail. In particular, in order to obtain the result that $\mathrm{T}_{\text {PARA }}$ is not committed to proper classes, we do not need to assume that it does not entail that there are proper classes. Rather, we take $\mathrm{T}_{\mathrm{PARA}}$ not to be committed to proper classes exactly because we recognize that any reason for taking $\mathrm{T}_{\mathrm{PARA}}$ to entail the existence of proper classes is straightforwardly question-begging.

Since the source of the problem of incomparability is the (realistic) possibility of disagreement, one might consider moving to some entailment relation weak enough to be incontestable in some sufficiently strong sense. For at least two reasons, this idea does not seem promising. Firstly, the resulting criterion would be pathetically and implausibly weak. Secondly, it does not seem as though in working out the implicit commitments of $\mathrm{T}_{\text {CLASS }}$ and $\mathrm{T}_{\text {PARA, }}$ we would, or should, only use principles that are uncontentious. For instance, even when comparing a classical theory with a paraconsistent one which rejects modus ponens, we should still use modus ponens in working out the commitments of the classical theory. In short, it seems that the appropriate thing to do is to use a theory's own logic in working out its implicit commitments. I conclude that the preceding considerations provide considerable evidence that any entailment-based criterion of implicit commitment employing any ordinary notion of entailment is incorrect.

\section{Peacock's Response}

Peacock is aware that (OCM) threatens to render ontological comparison between theories related like $\mathrm{T}_{\mathrm{U}}$ and $\mathrm{T}_{\mathrm{N}}$ impossible. With respect to an analogous case, he writes:

This problem case does not succeed in showing that the modal entailment conception of implicit commitment makes decision between theories on the basis

\footnotetext{
${ }^{18}$ As the subject matter of the theories we may take the question what set-like objects there are, so our question is which offers the more parsimonious answer to that question.
} 
of ontology impossible [...] However, the defender of [(OCM)] must make one concession: on this account it cannot be claimed that we can reach a final decision about the ontological commitments of metaphysical theories before reaching a decision about which theory is true. Our decisions between theories can only be made on the basis of what commitments we would recognize if we held the theory true [...] This is not an ideal situation, but it is good enough: at any rate, it does not prevent us from using the notion of implicit commitment to engage in comparison between theories on the basis of putative ontological cost. (Peacock 2011, p. 93.)

Peacock here points out that something else than choice between the theories on the basis of ontological commitment is possible even on (OCM), namely determining what we would recognize each theory as committed to were we to accept it, and deciding between the theories on that basis, i.e. on the basis of putative, not actual ontological cost. ${ }^{19}$ If this is to be consistent with the first sentence of the quoted passage, the phrase 'on the basis of ontology' cannot be read as a mere shorthand for 'on the basis of ontological commitment'. Instead, it must be interpreted in some way so that a choice between theories on the basis of putative ontological commitments can also qualify as a choice on the basis of ontology.

Note that nothing in Peacock's response to the cases of incomparability is specific to (OCM) as compared to other entailment-based criteria. One who prefers (OCL) to (OCM) might respond in exactly analogous fashion to the cases discussed in the previous section. For the sake of generality, I shall therefore at times discuss the proposal in a quasi-schematic way,

\footnotetext{
${ }^{19}$ Two exegetical remarks: Peacock sometimes seems to claim that (OCM) does not render choice between theories impossible in the cases at issue. Nothing he says seems apt to support this claim. The best interpretation of his view therefore seems to be the one assumed above, on which choice based on ontological commitment is conceded to be impossible, but in some sense choice based on ontology is nevertheless possible. Peacock also at times speaks of the commitments we would recognize were the theory true rather than the commitments we would recognize were we to think that the theory is true. I assume that the latter is intended. Generally speaking, whether a theory is true has no obvious implications for what we think about commitment, whereas what we take to be true does often have an impact on what we take theories to be committed to. Moreover, on standard accounts of counterfactuals, if a theory $\mathrm{T}$ is necessarily false, every instance of 'we would recognize $\mathrm{T}$ as committed to Fs if $\mathrm{T}$ were true' is vacuously true. If so, determining what we would recognize a theory as committed to were it true turns out problematic in a similar way as determining what it metaphysically entails.
} 
using 'entails' and related vocabulary as a stand-in for whatever notion of entailment one wishes to appeal to in one's account of ontological commitment.

Peacock's proposal can be factored into two independent components: a claim concerning how we can sensibly compare theories that are incomparable on (OCM), and the claim that (OCM), although not itself the basis on which to choose between theories like $\mathrm{T}_{\mathrm{UM}}$ and $\mathrm{T}_{\mathrm{NM}}$, is nevertheless the correct criterion of commitment. Both claims, we will see, are implausible. We consider the second claim first. In fact, we shall consider more generally the suggestion that we have a correct, entailment-based criterion of ontological commitment $C$, and a separate standard $S$ appropriate for choosing between theories incomparable on $C$. We assume that $S$ delivers intuitively plausible results. Note that $C$ has implications for the relative parsimony of theories even if it renders them incomparable: that the theories are incomparable only means that we cannot work out how they compare on $C$ in a non-questionbegging way, it does not mean that $C$ does not imply a comparative assessment. Now in some cases, $S$ and $C$ will come apart in what theory they recommend preferring on the grounds of parsimony. For definiteness, assume $S$ recommends preferring $\mathrm{T}_{\text {PARA }}$ to a version $\mathrm{T}_{\text {CLAsS }}$ of $\mathrm{T}_{\text {CLAss }}$ that postulates an entire hierarchy of classes, super-classes, super-duper-classes, etc., whereas $C$ delivers the opposite verdict. So we have that $\mathrm{T}_{\text {CLASS+ }}$ is more parsimonious than $\mathrm{T}_{\text {PARA. }}$ But then in what sense can $S$, in spite of recommending a theory over a competitor when the latter is more parsimonious than the former, nevertheless be a good standard for theory choice based on parsimony?

Perhaps someone might suggest that $S$, although not a perfect guide to comparative parsimony, is nevertheless a reasonably good guide, and perhaps the best we are in a position to apply in the relevant situations. But it is hard to see why $S$ should be considered even a reasonably good guide in application to the relevant cases. Certainly, the comparative assessment of $\mathrm{T}_{\text {PARA }}$ and $\mathrm{T}_{\text {CLASS }}$ that we obtain on $S$ is about as far off the assessments delivered by (OCM) or (OCL) as it could be - the latter after all count $\mathrm{T}_{\text {PARA }}$ maximally unparsimonious. The only alternative I see is to say that $S$, although not even a reasonable guide to comparative parsimony, reveals which of the theories considered is parsimonious in some other important respect. That is, we postulate a further theoretical virtue of hypothetical ontological parsimony, and say that, at least in cases that are ontologically incomparable, choice based on ontology ought to be based on comparative hypothetical parsimony. Moreover, we presumably couldn’t make do with just this one putative additional theoretical 
virtue, but would have to postulate 'hypothetical' versions of all the virtues that depend on what a theory implicitly says. Absent strong reasons for thinking that there are no viable alternatives to ordinary entailment-based criteria, this strategy seems desperate and ad hoc.

Let us then turn to Peacock's proposal for how to choose between the theories that are problem cases for (OCM). His idea is that we choose based on what we would recognize the pertinent theories as entailing the existence of, were we to accept them. That proposal seems indeed to avoid many of the problematic cases of incomparability: rarely is a premise of the form 'I would take $\mathrm{T}$ to entail the existence of Fs, were I to accept T' question-begging for a thinker comparing $\mathrm{T}$ with a rival theory. ${ }^{20}$ Nevertheless, it has quite implausible features. The crucial point is that what a thinker would recognize a theory as entailing were she to accept it therefore does not reliably track features of the theory relevant to its virtuousness. For what a given thinker would recognize a theory as entailing the existence of in general depends in part on irrelevant properties of the thinker, such as his independently held beliefs concerning entailment or his reasoning skills. Presumably, if I thought that sentences of the form 'there are some things which are arranged $F$-wise' entail corresponding instances of 'there are $F s^{\prime}$, I would recognize a theory containing such sentences as entailing the existence of Fs, were I to accept it, whereas if I thought the entailment did not obtain, I would not recognize the theory as having this entailment. Further, it may be that I would (not) recognize a theory as entailing a given claim merely because I am prone to make (what are by the lights of the theory) more or less subtle mistakes in drawing out its consequences.

What a thinker would recognize a theory as entailing were she to accept it therefore does not reliably track features of the theory relevant to its virtuousness. A natural idea for improving on Peacock's suggestion is to instead consider whether one should, or ought to recognize a theory as entailing the existence of Fs, were one to accept it. ${ }^{21}$ Plausibly, this condition can be interpreted in such a way that it is independent of a given thinker's properties. However, this modification creates other difficulties. In particular, it is hard to know what standards are appropriate for deciding whether one ought to accept a certain claim

\footnotetext{
${ }^{20}$ Although if $\mathrm{T}$ explicitly asserts a contradiction, and a rival $\mathrm{T}^{*}$ explicitly asserts that one strictly cannot accept theories containing outright contradictions (and that counterpossibles are vacuously true), it might be that unless $\mathrm{T}^{*}$ is false, every instance of ' $\mathrm{I}$ would take $\mathrm{T}$ to entail the existence of $F$ s, were $\mathrm{I}$ to accept $\mathrm{T}$ ' would be vacuously true, and many of these would thus probably be question-begging in the relevant context.

${ }^{21}$ Thanks here to an anonymous referee.
} 
were one to accept a given theory. Indeed, one might have hoped that this is something a criterion of commitment would tell one. ${ }^{22}$ Even setting this issue aside, we should still have to decide what notion of entailment is supposed to be invoked in the proposal. It therefore appears doubtful that the present suggestion constitutes progress, even as a stepping stone towards a more fully developed criterion. The next section tries a different route, taking as its starting point some of our earlier informal observations regarding cases like $\mathrm{T}_{\text {CLASS }}$ and $\mathrm{T}_{\text {PARA }}$.

\section{A Better Proposal}

I shall now develop and defend my preferred criterion of implicit commitment. The first step, carried out in §4.1, is to implement and defend the idea, suggested in the earlier discussion of $\mathrm{T}_{\text {CLASS }}$ and $\mathrm{T}_{\text {PARA }}$, that at least in cases of theories that include a logic, we should ask what the theories entail on their own logic, rather than what they actually logically entail. Two problems then remain. First, for some theories such as $T_{U}$ and $T_{N}$ above, there appears to be no such thing as their own logic, and nevertheless they seem to carry implicit commitments. Second, we often ascribe an implicit commitment to Fs to theories even though it is clear that the theory does not, on its own logic or on any other plausible logic, logically entail a sentence that means there are Fs - recall the example of $\{$ ' $\exists x x$ is a vixen' $\}$ intuitively being committed to foxes. The key element of my solution to these problems is the notion of an (overall) best explicit extension of a theory. I explain the relevant notion of an explicit theory in §4.2, and apply it to formulate my proposal for a criterion of commitment for ordinary theories in $\S 4.3$.

\subsection{Theories and their Logics}

The idea is that in choosing between theories including a logic, we ask what on their own logic they logically entail. Obviously, this will help avoid the problems of incomparability only if we can answer that question without begging the question against either theory. This is less obvious than it may appear at first glance.

\footnotetext{
${ }^{22}$ Thus, Peacock - quite plausibly, it seems to me - considers it a desideratum on a criterion of ontological commitment that it reveal 'what ontology we ought to adopt commensurate with some theory we do in fact accept' (Peacock 2011, 81).
} 
We can bring out the difficulty by asking just what the conditions are under which, on a given logic L, a set A (logically) entails a sentence S. Suppose we said that on a given logic L, A logically entails S iff L entails, in some ordinary sense, that A entails S. Then theories like Tclass and TPARA would still constitute problematic cases of incomparability. For generally speaking, a divergence between different accounts of logic extends to what these accounts themselves entail. For instance, the meta-theory advocated by Priest (1979) is just as inconsistent as some of his preferred object language theories. In classical logic it therefore entails absolutely everything, whereas by its own lights, it does not. So we had better not answer our question in this way. How, then, are we to answer it?

I think that at least on one natural and coherent way of understanding 'on L, A entails S', it is appropriately sensitive to what the logic in question says, so that instantiating it with, for example, Priest's inconsistent meta-theory does not yield a true sentence for arbitrary S. The very fact that we recognize that it is question-begging to reason classically in drawing inferences from that theory constitutes prima facie evidence for this, for it indicates that we have a conception of what it would mean to non-question-beggingly reason about a given logic. If so, it is this way of understanding the phrase which is pertinent in our context. ${ }^{23}$ Evidently, it will be at best very difficult to say anything helpful about how one can work out, for an arbitrary logic, what holds on it. For it is hard to see what one might say which is both more instructive than 'try not to beg any questions' and does not presuppose as valid some potentially contentious forms of reasoning, thereby reintroducing problematic cases of incomparability. In what follows, I will rely on the availability of a non-question-begging understanding of 'on L, A entails S'.

Assuming such an understanding, I see no reason to think that our suggestion yields implausible cases of incomparability. For absent general, substantive claims about the conditions under which on a given logic, a set of sentences entails another sentence, theories that are still rendered incomparable have to fairly explicitly make question-begging claims about what on another theory's logic, that theory logically entails. Thus, to obtain a theory incomparable with $\mathrm{T}_{\mathrm{PARA}}$, we have to include in the theory a claim such as that on $\mathrm{T}_{\mathrm{PARA}}$ 's logic, $T_{\text {PARA }}$ logically entails everything. I think that in such a case, it is plausible to say that

\footnotetext{
${ }^{23}$ What if there is no such coherent understanding of the phrase? In that case, I think we may plausibly conclude that the theories in question actually are strictly incomparable. If so, it does not count against our proposal that it renders them incomparable.
} 
the incomparability we obtain is genuine. (In addition, we may note that on the hypothesized reading of 'on L, A entails S', such a theory is also clearly false.)

I conclude that for theories which include a logic, we could improve on (OCL) by adopting instead the criterion

(OCL*) A theory $\mathrm{T}$ is ontologically committed to Fs iff

T includes a logic $\mathrm{L}$ such that on $\mathrm{L}$,

T logically entails some sentence that means there are Fs.

(Note that although (OCL*) still invokes the notion of logical entailment, it is not thereby an entailment-based criterion. For the role it gives to that notion is not the same role as that given to entailment in criteria of the form (OCE). ${ }^{24}$ )

But of course, we also ascribe implicit commitments to theories which do not include a logic, and we ascribe implicit commitment in excess of what, on any plausible logic, the theory in question logically entails. So (OCL*) does not fit the standards by which we actually evaluate theories. However, as I explain in the next subsection, it does fit the standards appropriate for evaluating what I will call explicit theories.

\subsection{Explicit Theories}

I begin by explaining my notion of an explicit theory, or more precisely: the notion of evaluating a set of sentences as an explicit theory. It is important here to bear in mind the

\footnotetext{
${ }^{24}$ Might it not be argued, however, that $\left(\mathrm{OCL}^{*}\right)$ is entailment-based after all, because the notion expressed by 'includes a logic L such that on L, it logically entails' is itself a notion of entailment? Suppose this is so. What is important for my purposes is merely that the latter notion is different in kind from the notions of logical / analytical / metaphysical / ... entailment, which seems undeniable. If nevertheless, all the notions are notions of entailment, I may simple use different terminology to draw the intended distinction. Thus, we may call a notion of entailment internal iff, for some notion of entailment we shall express by 'entails*', the notion is defined by: T entails sentence $S$ iff $\mathrm{T}$ includes a theory of entailment* on which $\mathrm{T}$ entails* $S$, and call a notion of entailment external iff it is not internal. Then the notion of entailment on which my (OCL*) is based is an internal notion of entailment, whereas the notions on which (OCL) et al are based are all external notions of entailment. My proposal, therefore, while entailment-based, is not external-entailment-based, and this feature of the proposal is essential for the solution to the problem of incomparability it provides. Readers wishing to count the notion of a theory's including a logic on which it entails some sentence a notion of entailment may therefore simply qualify relevant occurrences of 'entailment-based' in the subsequent sections by 'external'.
} 
point that a theory, i.e. a set of sentences, is not good or bad simpliciter; it is good or bad only in some respect or other. The same set of sentences may be good as an example of a set of very long sentences, but bad as a theory of composition. In theory choice, then, we ask of the relevant sets how good they are as theories. Of course, we do not, and cannot sensibly compare any old pair of theories. For instance, it is hard to attach any sense to the question whether Peano Arithmetic is a better theory than Modal Realism. What we sensibly compare are theories which are in competition with one another, which requires that they have a common subject matter. More precisely, then, what we ask in theory choice is how good certain sets of sentences are as theories of some particular subject matter.

In evaluating a set of sentences as a theory (of some subject matter), we evaluate it as, roughly speaking, a response to a request for a collection of sentences that accurately answers, explicitly or implicitly, as many questions pertaining to its subject matter as possible. A set of sentences is a good theory to the extent that it is a good response to that request. But of course, we could also evaluate a set of sentences as a response to a slightly different kind of request. Say that a theory quasi-explicitly answers the question whether $p$ just in case it includes an account of logical consequence on which it logically entails either a sentence meaning $p$ or a sentence meaning not- $p .^{25}$ Then we could evaluate a set of sentences as, roughly, a response to a request for a collection of sentences that accurately and quasiexplicitly answers as many questions pertaining to a given subject matter as possible. I will say that to thus evaluate a set of sentences is to evaluate it as an explicit theory (of the relevant subject matter).

For sets of sentences considered as explicit theories, I contend, the criterion of commitment envisaged above is adequate (' $\mathrm{X}$ ' is for 'eXplicit'). ${ }^{26}$

\footnotetext{
${ }^{25}$ This stipulation has the admittedly odd-sounding consequence that $\{$ ' $p$ ' $\}$ does not quasi-explicitly answer the question whether $p$, since it does not include an account of logical consequence. We could easily avoid this result by adding 'it includes a sentence meaning $p$ or meaning not-p' as a second disjunct to the definiens. Since it is not my aim to capture or approximate any ordinary meaning of 'quasi-explicit', but use the term merely as a partly suggestive label for a purely technical notion, I shall stick to the definition above.

${ }^{26}$ Strictly speaking, the left-hand side of the biconditional should be something like 'a set of sentences $\mathrm{T}$ is ontologically committed to Fs qua explicit theory'. I shall keep using the less cumbersome formulation above which may be read as shorthand for the more strictly adequate reformulation.
} 
On (OCX), some sets of sentences including, for instance, the sentence ' $(\exists x x$ is a number) \& ( $\exists x x$ is a number)', are not committed to numbers when considered as explicit theories. Properly interpreted, this is a plausible result. There are two cases to consider: first, a set including ' $\exists x x$ is a number) \& ( $\exists x x$ is a number)' may not quasi-explicitly answer the question whether there are numbers, thereby avoiding the commitment, or second, it may quasi-explicitly answer the question negatively. Either way, it is plausible that, qua explicit theory, it is not committed to numbers. Note, moreover, that any such set is bound to be an overall very poor explicit theory, because it is bound to score very low with respect to theoretical virtues other than ontological parsimony. One of the most important criteria for how good some set is as a theory of some subject matter is how comprehensive it is with respect to that subject matter. Roughly speaking, that is a matter of how many questions pertaining to its subject matter it answers. For explicit theories, then, their comprehensiveness is determined by how many questions pertaining to their subject matter they quasi-explicitly answer. So in the first case, the explicit theory is objectionably lacking in comprehensiveness with respect to whatever we may plausibly consider its subject matter, and in the second, it evidently fails to be compatible with things we know, such as that conjunction elimination is valid. This consideration illustrates how the importance of theoretical virtues other than ontological parsimony blocks obvious cheap strategies for avoiding ontological commitment by the standards of (OCX).

\subsection{Ordinary Theories}

We now return to the question of how ordinary, i.e. non-explicit theories are to be compared, and what they are to be counted as implicitly or explicitly committed to. In one way, the consideration of explicit theories has not gotten us any closer to an answer to that question: we have managed to say something plausible about them simply because here, by stipulation, the question of implicit commitment has a straightforward answer. Nevertheless, our discussion makes salient some strategies for answering our question which, I shall argue, turn out to be much more plausible than those considered so far. 
Note first that the question what claims an ordinary theory is explicitly or implicitly committed to is roughly equivalent to the question how we have to enrich the theory to obtain an explicit theory that carries just the commitments of the ordinary one. Our earlier criteria can accordingly be seen as providing answers to that question. On (OCM), we have to enrich it by all and only those sentences expressing claims that are metaphysically entailed by the original theory, and on (OCL) we have to enrich it by all and only its logical consequences. The problem was that the question what these sentences are cannot always be answered without begging the question.

Our standard for comparing explicit theories suggests a further possibility: a given theory of a subject matter $\mathrm{X}$ is to be enriched so that what we obtain is the best explicit extension of the theory, where 'best' means best by the overall standards of theory choice for explicit theories, including corresponding standards of comprehensiveness, elegance, compatibility with what we know alongside the standard for ontological parsimony provided by (OCX). On at least two counts, that suggestion seems preferable to a counterpart which considers the extensions of theories by their actual logical consequences. First, since determining what view of logic yields the overall best explicit theory when combined with a given theory does not require answering the question which view of logic is correct, the proposal avoids the implausible incomparability results of its counterpart. Second, even setting aside difficulties in comparing such pairs of theories, the proposal has greater plausibility. Given a partial account of some subject matter, it seems, what we should ask, and generally do ask, is how attractive it is on the best way of developing it into a complete account of its subject matter. If we find that the best way of developing it combines it with a view of logic that we reject, then we consider the result of so extending it. We may then find that the view of logic in question is sufficiently implausible that the theory can be rejected, or that the overall package is sufficiently attractive to merit further consideration. But what we do not, and should not, do, is to simply evaluate the theory in terms of its consequences on the view of logic we independently consider correct, as we should were we trying to apply the alternative criterion. Analogous points apply with respect to criteria based on other notions of entailment.

For illustration of the proposal, consider as a toy example the following theories of the Liar: $\{$ 'the Liar sentence is true and not true'\}, and \{'the Liar sentence does not express a proposition'\}. On an account of commitment which considers extensions by actual logical 
consequences, the former theory is maximally unattractive for it logically entails everything. In addition, the two theories are incomparable, for we only obtain this result on the basis of classical logic, which begs the question against the former theory. The present approach, in contrast, has us ask what the best fully explicit theories of the Liar paradox are that incorporate our mini-theories. While this is a very difficult task, we can go about solving it without begging the question against either theory. One such best explicit extension will presumably be close to Priest's view in endorsing a paraconsistent logic to accommodate the contradictions accepted as true. The other may be a classical view employing some criterion of non-circularity for a sentence to express a proposition. We can then try to measure the comparative theoretical costs of adopting paraconsistent logic and of taking a more restrictive view on when a sentence expresses a proposition. Crucially, this seems an appropriate and constructive way of going about comparing the theories, or more realistic counterparts of them, and it seems to be how the pertinent debates are actually conducted.

How exactly should we state the envisaged criterion of commitment? In its simplest form, the idea would be to identify the commitments of an ordinary theory with those of its best extension, considered as an explicit theory. It is not clear, however, that there will always be a unique best extension of a given theory. We can do without this assumption by instead considering what each of the theory's best extensions is committed to. A second minor difficulty is that our standard for comparing explicit theories is not absolute, but relative to a given subject matter. That is, given some ordinary theory, we can speak only of all or some of its best extensions relative to a given subject matter, not of its best extension simpliciter. Call an extension of a theory $\mathrm{T}$ an X-best explicit extension of $\mathrm{T}$ if no extension of $\mathrm{T}$ is a better explicit theory of $\mathrm{X}$. The most plausible standard for Ontological Commitment of Ordinary theories that we can straightforwardly construct on that basis is then as follows:

(OCO) An ordinary theory T is ontologically committed to Fs qua theory of X iff every X-best explicit extension of $\mathrm{T}$ is ontologically committed to Fs.

To appreciate the distinctive features of this proposal, it is instructive to reflect on its relation (OCL), which, roughly, counts a theory T committed to Fs iff T logically entails that there are Fs. (OCO) can be seen as obtained from (OCL) through a relativization to subject matter combined with two important replacements: first, a replacement of the appeal to $\mathrm{T}$ on the right-hand side by an appeal to the best explicit extensions of $\mathrm{T}$, and second, a replacement of 
the appeal to the notion of logical entailment by an appeal to the notion of a theory's logical entailments according to the theory's own logic.

Ordinarily, of course, we do not relativize ascriptions of commitment to a subject matter. Plausibly, however, in ordinary contexts, it is sufficiently clear what the relevant theory is supposed to be a theory of. The commitments we ascribe to it in such contexts can then be identified with the commitments we would ascribe to it on (OCO), relative to the contextually determined subject matter. ${ }^{27}$ I know no example of an intuitively very plausible ascription of (a lack of) commitment that contradicts this identification.

As for a standard of comparative parsimony, there are now two salient options. One is to say, just as before, that a theory $T_{1}$ is more parsimonious than another $T_{2}$ just in case $T_{1}$ carries less substantial ontological commitments than $T_{2}$. The other is to say that $T_{1}$ is more parsimonious iff every best extension of it is more parsimonious than every best extension of $\mathrm{T}_{2}$. The conditions are not obviously equivalent when one or both theories have multiple best extensions $^{28}$, but it seems plausible that for any intuitively clear examples of greater comparative parsimony, they will deliver the same, intended results.

Note that (OCO) does of course render some pairs of theories incomparable. Trivial examples are provided by theories that explicitly make competing claims about what sentences their rivals' best extensions include or fail to include. It seems plausible, however, that these cases at least are cases of genuine incomparability, and that few if any realistic and interesting pairs of theories are thus related. More interesting cases arise when, roughly speaking, one theory properly extends another by some implausible claim. Thus, let $\mathrm{T}$ be $\left\{{ }^{`} \exists x\right.$

\footnotetext{
${ }^{27}$ Note that the subject matter-relativity of (OCO) may even help explain some data concerning ascriptions of commitment. For instance, in most contexts, most philosophers will be happy to say that set theory is ontologically committed to abstract objects. Nevertheless, it also seems tempting to say that as a piece of pure mathematics, standard set theory does not carry any distinctive metaphysical commitments. (OCO) allows us to explain this kind of variation as a variation in what subject matter is at issue - pure mathematics in the one case, and a more comprehensive, partly metaphysical account of sets in the other.

${ }^{28}$ Suppose that the relevant differences in commitment are as follows. The unique best extension of $T_{1}$ is committed to Fs, whereas one best extension of $\mathrm{T}_{2}$ is committed to $G$ s, and the only other best extension of $\mathrm{T}_{2}$ is committed to Hs. Then on the first condition, we have to ask whether a commitment to Fs is more or less substantial than a commitment to Gs-or-Hs. (This assumes that both best extensions are committed to Gs-or-Hs, which is guaranteed if both include a logic validating or-introduction.) On the second condition, we have to ask how a commitment to Fs compares to both a commitment to Gs and a commitment to Hs.
} 
$x$ is a set' $\}$, and assume that its best extension is committed to abstract objects. It seems that we cannot non-question-beggingly obtain this result when comparing $\mathrm{T}$ with the result of adding to it a sentence claiming sets to be concrete. Again, it seems to me that such cases involve genuine incomparability. It really does seem that when one ascribes to $\mathrm{T}$ a commitment to abstract objects, one has implicitly ruled out that sets might be concrete.

Moreover, this kind of incomparability case does not seem very problematic. For we can of course adequately compare an orthodox metaphysical account of sets with a heterodox one on which sets are concrete - it is only that we must include in the former a statement of the view of sets as abstract. Roughly speaking, the general lesson afforded by our case is that to be able to assess competing views by means of Occam's Razor or other general principles of theory choice, we must apply these principles to sets of sentences that quasi-explicitly articulate at least those components of the views in which they diverge. Let us say that two ordinary theories compete just in case they are so related. It seems appropriate then to restrict Occam's Razor and its ilk to pairs of competing theories.

That being said, it is not obvious that on the present approach, there is much reason to apply principles like Occam's Razor to ordinary theories. For suppose I consider two competing ordinary theories. To see which is more parsimonious, I must find out what their ontological commitments are, and so I have to work out what their best extensions are. But once I have done that, the appropriate thing to do may seem to be to ask which of these is preferable by the overall standards for explicit theories: What is the point in returning, as it were, to the ordinary theories, to work out which of them is favoured by the various principles for ordinary theories? Rather, we should simply have an overall standard for theory choice between competing ordinary theories in terms of the relative preferability of their explicit extensions.

I have no need to take a stand on this issue here. We may note though, that even if we proceed in this fashion, we still have some use for (OCO). For since philosophers often do to ascribe implicit ontological commitments to ordinary theories, and make judgements of comparative parsimony concerning them, we had better have a way of making sense of what such ascriptions and comparative judgements might reasonably be getting at. (OCO) yields such an interpretation. 
One might further be tempted to object to my proposal that it makes comparison of theories impossibly difficult. Roughly, to determine what a given theory is committed to, we now need to figure out what its best explicit extension quasi-explicitly says. But surely in most cases, we simply don't know which of the theory's infinitely many extensions is best! The latter point may be conceded immediately, but all that follows is that in most cases, we won't be in a position to specify completely and exactly the commitments of a given theory. There was never reason to think that we should in general be in a position to do that. Nevertheless, in many cases we know enough about what the best explicit extension of a given theory looks like to know all or most of the theory's distinctive commitments. At any rate, it seems very implausible that there are cases where we know that a given theory is committed to Fs, but do not know whether its best explicit extension quasi-explicitly says that there are Fs. If there aren't, there is no reason to think my proposal makes knowledge of a theory's commitments harder to come by than it actually is.

\section{Conclusion}

Prima facie, in theory choice we take into account what a given theory merely entails, in some suitable sense, as well as what the sentences making up the theory explicitly say. Indeed, many proposed principles of theory choice, and in particular most criteria of ontological commitment that have been put forth in the literature, incorporate this idea. Unfortunately, these principles behave implausibly with respect to theories that disagree on matters of entailment: they predict that rational and non-question-begging theory choice, based on the usual theoretical virtues, is impossible in such cases. In many of the relevant cases, this seems clearly wrong. After considering and rejecting an attempt by Howard Peacock to defend an entailment-based criterion of ontological commitment in view of this difficulty, I developed an alternative proposal which, I argued, fits much better with how we actually, and reasonably, choose between theories, even when these differ over issues as basic as logical consequence. On this proposal, what we consider in theory choice in addition to the explicit content of a theory - its implicit commitments - is a matter not of what the theory entails, but of what the best explicit theory extending it quasi-explicitly says. ${ }^{29}$

\footnotetext{
${ }^{29}$ The earliest predecessor to this paper was written just over five years ago. Very many people provided very valuable feedback, criticism, and encouragement over the various stages of development the paper has since
} 


\section{References}

Azzouni, J. (2007). 'Ontological Commitment in the Vernacular'. Noûs 41, 204-26

Cartwright, R. (1954). 'Ontology and the Theory of Meaning’. Philosophy of Science 4, 31625

Devitt, M. (1980) ““Ostrich Nominalism” or “Mirage Realism”?’. Pacific Philosophical Quarterly 61, 433-9

Fine, K. (2009). 'The Question of Ontology'. In Chalmers et. al (eds.), Metametaphysics (Oxford UP), 157-77

Jackson, F. C. (1989). 'A Puzzle about Ontological Commitment'. In Heil (ed.): Cause, Mind and Reality (Dordrecht, Kluwer), 191-200

Michael, M. (2008). 'Implicit Ontological Commitment'. Philosophical Studies 141, 43-61

Oliver, A. (1996). 'The Metaphysics of Properties’. Mind 105, 1-80

Parsons, T. (1967). 'Extensional theories of ontological commitment'. Journal of Philosophy 64, 446-450

Peacock, H. (2011) 'Two Kinds of Ontological Commitment'. The Philosophical Quarterly 61, No. 242, 79-104

Priest, G. (1979). ‘The Logic of Paradox’. Journal of Philosophical Logic 8(1), 219-41

Priest, G. (2006). In Contradiction ( $2^{\text {nd }}$ edition). Oxford: Clarendon

Quine, W. V. O. (1961). 'Notes on the Theory of Reference'. In his From a Logical Point of View ( $2^{\text {nd }}$ ed., New York: Harper Torchbooks), 130-8

Rayo, A. (2007). 'Ontological Commitment'. Philosophy Compass 2, 428-44

Searle, J. (1969). Speech acts. Cambridge: Cambridge University Press

Simons, P. M. (1997). 'Higher-Order Quantification and Ontological Commitment'. Dialectica 51(4), 255-71

Williamson, T. (2007). The Philosophy of Philosophy. Oxford : Blackwell.

undergone. I thank all of them, and especially John Divers, Joseph Melia, Howard Peacock, Benjamin Schnieder, Moritz Schulz, Jason Turner, Robbie Williams, as well as three anonymous referees. 